OPEN

SUBJECT AREAS:

COORDINATION

CHEMISTRY

ORGANIC-INORGANIC NANOSTRUCTURES

NANOPARTICLES

Received

7 May 2014

Accepted

9 June 2014

Published

27 June 2014

Correspondence and requests for materials should be addressed to

X.Z.Y. (youxz@nju. edu.cn) or X.X.X. (xuxx@nju.edu.cn)

\section{Colloidal Nanocrystals Fluoresced by Surface Coordination Complexes}

\author{
Guan Wang' , Jianwei Ji' 'Xinwen Zhang ${ }^{2}$, Yan Zhang ${ }^{3}$, Qiangbin Wang ${ }^{3}$, Xiaozeng You' \& Xiangxing Xu'
}

'State Key Laboratory of Coordination Chemistry, School of Chemistry and Chemical Engineering, Nanjing National Laboratory of Microstructures, Nanjing University, Nanjing 210093 , P. R. China, ${ }^{2}$ Key Laboratory for Organic Electronics \& Information Displays, Institute of Advanced Materials, Nanjing University of Posts and Telecommunications, 9 Wenyuan Road, Nanjing 210046, P. R. China, ${ }^{3}$ Division of Nanobiomedicine and i-Lab, Suzhou Institute of Nano-Tech and Nano-Bionics, Chinese Academy of Sciences, Suzhou, 215123 , P. R. China.

Colloidal Nanocrystals (NCs) with fluorescence originating from surface complexes are successfully prepared. The components of these NCs range from insulator, semiconductor to metal, with either pure phase, doped or core/shell structures. The photoluminescence of these NCs can be reversibly tuned across the visible to infrared spectrum, and even allow multi-color emission. A light emitting device is fabricated and a new in vivo cell imaging method is performed to demonstrate the power of this technology for emerging applications.

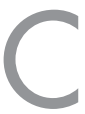
olloidal nanocrystals (NCs) are finding increasingly varied applications ranging from electronics ${ }^{1-5}$ to energy $^{6-8}$ and to biology ${ }^{9-11}$. To achieve the exquisite control over the properties of NCs, one approach is to modify surfaces of NCs with organic or inorganic molecules, which are known as ligands. The use of ligands is the favored tool for the control of the growth of colloidal NCs, tailoring the morphology ${ }^{12-14}$, enhancing the dispersibility and stability ${ }^{11}$, facilitating assembly into artificial molecules or patterns ${ }^{1,15-17}$, and inducing interactions such as exciton coupling ${ }^{18}$, energy or electron transfer between the core and the surface ligands of a $\mathrm{NC}^{19-22}$.

Structurally, a colloidal NC is similar to a typical coordination complex, containing the inner core and the outer ligands. However, the method to study the coordination complex can hardly be applied to a whole colloidal NC directly due to the large number of atoms consisted of a colloidal NC core, the uncertainty of the NC structure in atom level precise, and also due to the diversity of size and/or shape of the NCs even prepared in the same batch. For these reasons, differing from attempting to study a colloidal nanocrystal as one big coordination complex system, and also differing from the traditional 'Ligand on NC' model, a 'Surface Coordination Complex on NC' model is adopted here. That is, a NC surface metal ion (M) together with its covalently bonded atoms and surface coordination ligand (L) are regarded as a localized system the surface complex. The rationality can be seen from the view of coordination chemistry, where metal ions and ligands are treated as an integrated system to interpret the properties of compounds. The molecular tailorability of the coordinated ligands and the advances in NC synthesis make it possible to create various colloidal NCs with functional surface complexes. Here we report the application of this concept to prepare a series of NCs with fluorescence originating from surface complexes by the simple ligand exchange approach (Fig. 1). The cores of these fluorescent NCs cover a wide range of nanomaterials including insulator (e.g., $\mathrm{Al}_{2} \mathrm{O}_{3}, \mathrm{MgO}$ ), semiconductor (e.g., $\mathrm{ZnS}, \mathrm{CdSe}, \mathrm{TiO}_{2}, \mathrm{ZrO}_{2}$ ) to metal NCs (e.g., Au), with either pure phase, doped (e.g., lanthanide doping) or core/shell structures (e.g., CdSe/CdS, Au/ZnS), exhibiting the potential possibilities in function combinations. As is well known that the fluorescence of coordination complexes can be categorized into four types ${ }^{23}$ : for type-A fluorescence, the emission comes from the transition of $\mathrm{L}^{*} \rightarrow \mathrm{L}\left(\mathrm{L}^{*}\right.$ is the excited state of the ligand $\mathrm{L}$ ), where metal ion $\mathrm{M}$ behaves as the indispensable perturbation; for type-B fluorescence, the emission comes from the transition of $M^{*} \rightarrow M\left(M^{*}\right.$ is the excited state of the metal ion $\mathrm{M}$ ), where L may affect as an "antenna", which absorbs and transfers energy to M; there are also $\mathrm{L}^{*} \rightarrow \mathrm{M}$ and $\mathrm{M}^{*} \rightarrow \mathrm{L}$ fluorescence, in which charge transfer is involved. Due to the complexity of the later two types of fluorescent that may involve the charge transfer not only between $L / L^{*}$ and $M / M^{*}$, but also to the NC core, this paper focuses on the former two types of fluorescent surface coordination complexes on colloidal NCs. 


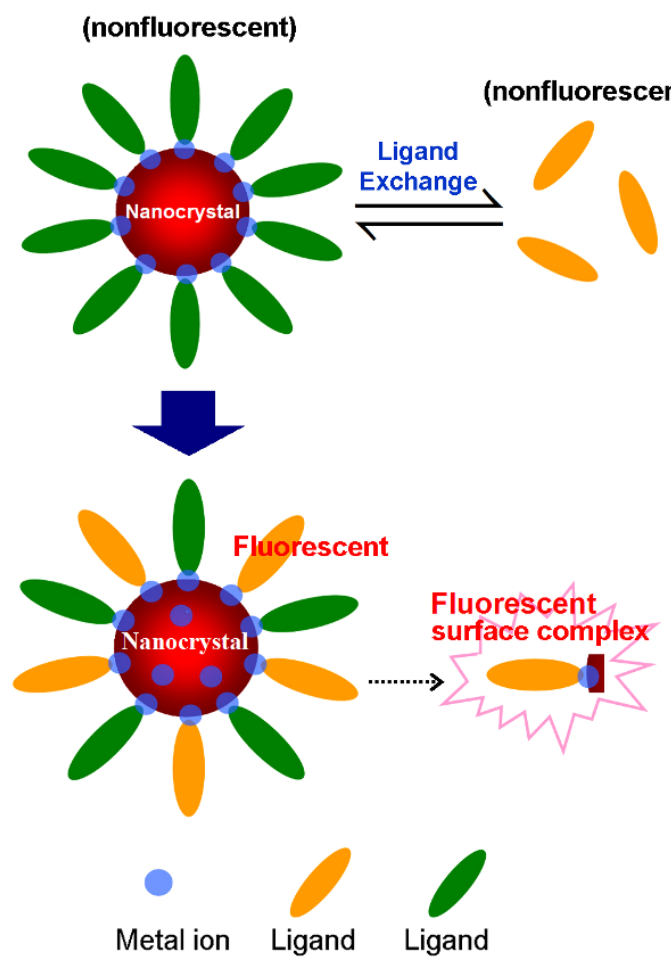

Figure 1 Schematic illustration of the ligands exchange strategy that brings fluorescence to a colloidal NC via surface complexes.

\section{Results and Discussion}

Colloidal NCs with Type-A Fluorescent Surface Coordination Complexes. Inspired by type-A fluorescent coordination compounds formed of the type $\mathrm{MQ}_{\mathrm{x}}(\mathrm{M}=\mathrm{Al}, \mathrm{In}, \mathrm{Zn}, \mathrm{Mg}$ or Li etc; $\mathrm{x}=3,2$, or 1 according to the valence state of $\mathrm{M} ; \mathrm{Q}=8$ hydroxyquinoline), a series of colloidal NCs with type-A fluorescent surface coordination complexes were synthesized, by using 8hydroxyquinoline as ligands and with varied morphologies of dot, flake or wire (Fig. 2, Fig. S1, S2). The photoluminescence (PL) spectra of NCs that contain different metals ions, but with the same surface ligand 8-hydroxyquinoline, were found to be diverse. For example $\mathrm{MgO}-\mathrm{Q}$ NCs emits blue light, $\mathrm{Al}_{2} \mathrm{O}_{3}$-Q blue-green and $\mathrm{ZnS}$-Q green (Fig. $2 \mathrm{j}, \mathrm{k}$ ). The crystalline structures of the NCs are preserved after the surface ligands exchange of 8-hydroxyquinoline with the original oleic acid and/or oleylamine ligands, as verified by Transmission Electron Microscopy (TEM, e.g., Fig 2c and Fig. S1b) and X-ray diffraction (XRD, e.g., Fig. S1c). The existence of surface complexes was supported by photoluminescence, UV-vis absorption, Fourier transform infrared spectroscopy, and excitation spectrum measurements. The remaining oleic acid and/or oleylamine ligands help maintain of the dispersibility of the NCs in nonpolar solvent such as cyclohexane, chloroform or toluene ${ }^{18}$.

Colloidal NCs with Type-B Fluorescent Surface Coordination Complexes. Colloidal NCs with type-B fluorescence were obtained for NCs containing lanthanide ions of the surface coordination complexes, for example, by using 2-thenoyltrifluoroacetone (TTA) - a typical ligand for fluorescent lanthanide complexes coordinate to $\mathrm{Eu}, \mathrm{Nd}, \mathrm{Yb}$ or Er doped $\mathrm{ZrO}_{2}, \mathrm{TiO}_{2}$ and $\mathrm{ZnS} \mathrm{NCs}$ (Fig. 2d-i,l,m, Fig. S3). It means that, rather than searching for efficient host crystals, the surface complex approach allows the component, crystal type and structure of the NC core to become less important to obtain the strong lanthanide ion fluorescence. Typically, TTA-coordinated $\mathrm{Eu}, \mathrm{Nd}, \mathrm{Yb}$ or Er doped NCs result in more than 50 times higher intensity of the lanthanide-based photoluminescence than that of the as synthesized NCs before TTA coordination (e.g., Fig. 2m).
Multi-color Emission and PL Tunability. For multiple lanthanide ion co-doped NCs, e.g. Eu, $\mathrm{Yb}, \mathrm{Nd}$ and Er co-doped $\mathrm{ZrO}_{2}$ or $\mathrm{TiO}_{2}$ NCs, when coordinated with TTA, the photoluminescence spectrum presents multi-color emissions ranging from visible to the near infrared region (Fig. 3a-d). For the advantage of the simplicity, this multi-color fluorescence strategy beats the molecular design strategy to synthesize heteronuclear complexes with centre metal ions of more than 2 elements. Since the surface complex model suggests that tunable photoluminescence of the surface complex can be anticipated according to that of the corresponding free standing complexes, by tuning the structure of coordination ligand of 8-hydroxyquinoline, 2-(2-(9H-fluoren-2-yl)vinyl)-8-hydroxyquinoline $(\mathrm{QF})$ was synthesized. The coordination complex $\mathrm{Zn}(\mathrm{QF})_{2}$ emits yellow fluorescence with peaks centered at $\sim 535 \mathrm{~nm}$ (Fig. S4); accordingly, when QF is applied to the $\mathrm{ZnS}$ nanocrystals, it gives expected yellow fluorescence. Therefore, distinct fluorescence emissions can be selectively activated by using appropriate coordinated ligands binding to the same NCs. Fig. 3e,f show the photoluminescence images and spectra of Eu:ZnS based type-A or type-B fluorescent colloidal NCs, exhibiting green $(\sim 498 \mathrm{~nm})$, yellow $(\sim 545 \mathrm{~nm})$ or red $(\sim 617 \mathrm{~nm})$ emission where ligands of $\mathrm{Q}$, QF or TTA are coordinated, respectively. This series of fluorescence can be reversibly switched between each other simply by ligands exchange, which is rarely seen for heteronuclear complexes.

Fluorescent Noble Metal NCs. This NC fluorescent strategy can also be applied to noble metal NCs. Noble metal NCs and their alloy NCs nowadays attract interests for their application potentials in biology, catalysis and electronic industry. To combine the optical property with the noble metal NCs, efforts have been attempted on developing heterostructured nanomaterials. However, due to the intimate contact of the metal and semiconductor, which promotes charge separation across the interface, severe PL quenching was encountered, such as in $\mathrm{Au}-\mathrm{CdSe}, \mathrm{Au}-\mathrm{PbSe}, \mathrm{Au}-\mathrm{PbS}$, FePt-CdS nanoparticles ${ }^{24,25}$. Silica coated dye doping $\left(\mathrm{Ag} / \mathrm{SiO}_{2} / \text { dye }\right)^{26}$ and hybrid superstructures ( $\mathrm{Au} /$ $\mathrm{SiO}_{2}$ /quantum dot) ${ }^{27}$ are two reported successful strategies toward fluorescence. Here the noble metal NCs functionalized with fluorescent surface complexes are demonstrated. When europiumdoped gold NCs (Eu:Au NCs) are coordinated with TTA, significant photoluminescence from the europium surface complex is observed (Fig. 4a,b and Fig. S5a). Another approach to construct noble metal NCs with fluorescent surface complexes is by consideration of core/shell- or hetero-structured NCs. As an example, core/shell structured Au/ZnS-Q NCs were prepared, which have blue-green photoluminescence (Fig. 4c,d and Fig. S5b). These two strategies presented here successfully achieved the goal of combining photoluminescence properties with non-fluorescent noble metal NCs, overcoming the quenching effect that would be encountered in metal-semiconductor heterostructures.

Discussion on Type-A Fluorescent Surface Coordination Complexes. In a NC system with type-A fluorescent surface complexes, the physic properties of the NC core contribute non-negligible effects. For example, two indium-based NCs, InOOH-Q and $\mathrm{In}_{2} \mathrm{~S}_{3^{-}}$ $\mathrm{Q}$, respectively emit green light and are non-emissive. The energy level alignment model is used trying to interpret the fluorescence behaviors of NCs with type-A surface complexes. Fig. 5a shows the proposed energy level alignment for the $\mathrm{ZnX}-\mathrm{Q}(\mathrm{X}=\mathrm{O}, \mathrm{S}$ or $\mathrm{Se})$ system. The energy level of '-XZnQ' $(\mathrm{X}=\mathrm{Se}, \mathrm{S}$ or $\mathrm{O})$ is approximately estimated from that of the $\mathrm{ZnQ}_{2}{ }^{28}$. The conduction band of $\mathrm{ZnS}$ NC is above the lowest unoccupied molecular orbital (LUMO) level of the surface complex '-SZnQ', and the valence band is lower than the highest occupied molecular orbital (HOMO) level of the surface complex. This type of energy level alignment will contribute to the confinement of the surface complex exciton in the surface complex layer, inducing enhanced photoluminescence. Similar energy alignment facilitates the photoluminescence for either 

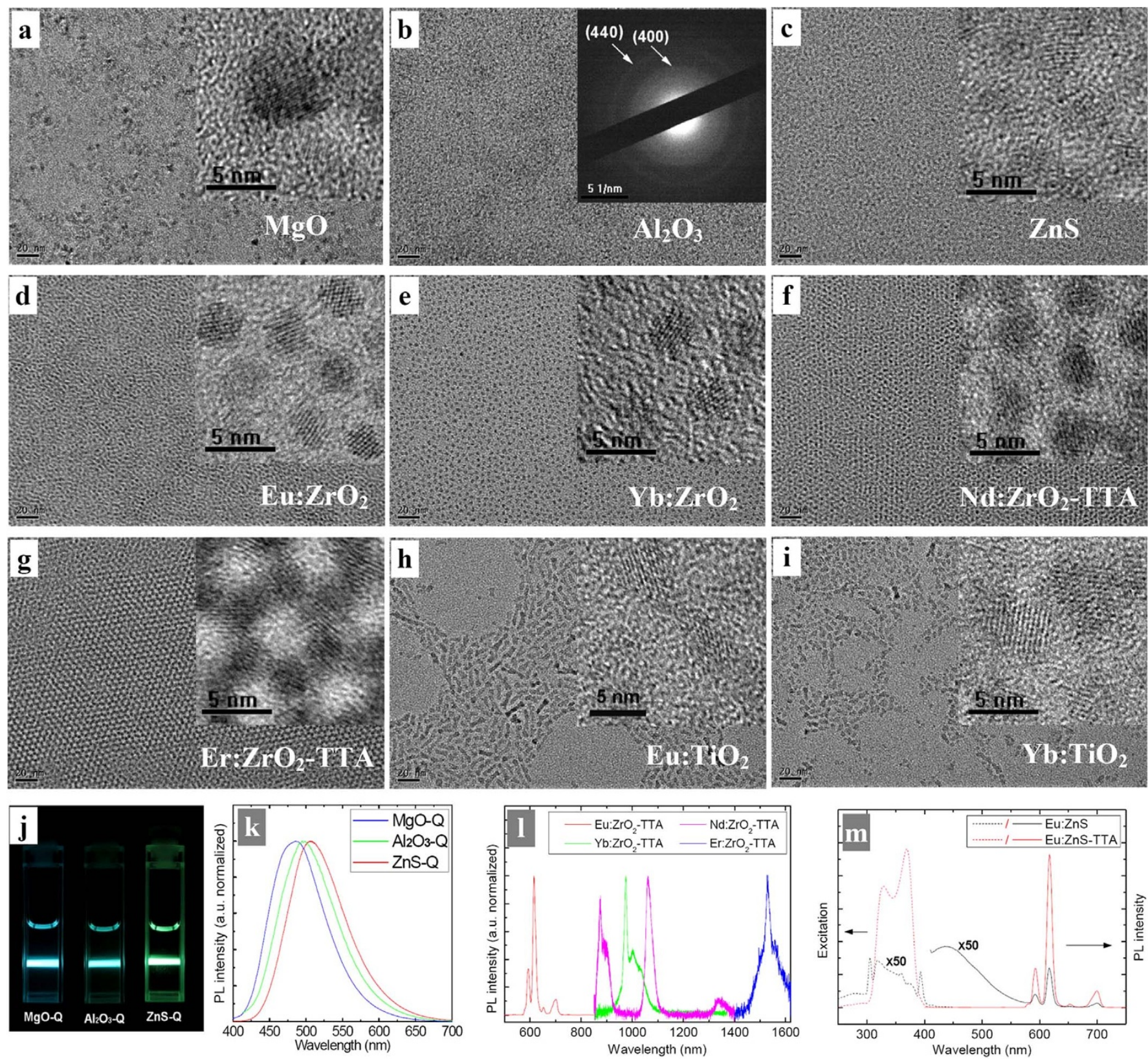

Figure 2 Colloidal nanocrystals fluoresced by surface coordination complexes. TEM images of (a) $\mathrm{MgO}$, (b) $\mathrm{Al}_{2} \mathrm{O}_{3}$ (inset is the electron diffraction pattern), (c) ZnS, (d) Eu:ZrO $\mathrm{Zr}_{2}$, (e) $\mathrm{Yb}: \mathrm{ZrO}_{2}$, (f) Nd:ZrO $2-\mathrm{TTA}$, (g) Er:ZrO $2-\mathrm{TTA}$, (h) Eu: $\mathrm{TiO}_{2}$ and (i) $\mathrm{Yb}_{2}$ TiO $\mathrm{Z}_{2}$ NCs. (j) The photoluminescence images and $(\mathrm{k})$ photoluminescence spectra of the $\mathrm{MgO}-\mathrm{Q}, \mathrm{Al}_{2} \mathrm{O}_{3}-\mathrm{Q}, \mathrm{ZnS}-\mathrm{Q}$ NCs. (1) Photoluminescence spectra of $\mathrm{Ln}: \mathrm{ZrO} \mathrm{O}_{2}-\mathrm{TTA}(\mathrm{Ln}=\mathrm{Eu}, \mathrm{Yb}, \mathrm{Nd}$ or Er) NCs. (m) Photoluminescence and photoluminescence excitation spectra of Eu:ZnS and Eu:ZnS-TTA NCs (Excitation: 360 nm; Emission: 617 nm), measured with the same NC concentration. ((a) - (i) scale bar $20 \mathrm{~nm}$, inset scale bar $5 \mathrm{~nm}$. (j) - (m) Excitation: $360 \mathrm{~nm}$; solvent: cyclohexane).

doped NCs such as $\mathrm{Zn}: \mathrm{ZrO}_{2}-\mathrm{Q}$ (Fig. S6) or insulators such as $\mathrm{Al}_{2} \mathrm{O}_{3}$ and $\mathrm{MgO} \mathrm{NCs}$ which have even larger bandgaps. The validity of the model obtained more support by studies of ZnSe-Q and ZnO-Q NCs. As the conduction band of $\mathrm{ZnSe}$ or $\mathrm{ZnO} \mathrm{NCs}$ is lower than the LUMO level of the corresponding surface complex, and the valence band is lower than the HOMO level, the excited electron of the surface complex would transfer to the NC while the hole is confined within the surface complex. This charge separation promotes non-radiative recombination thus the NCs are nonfluorescent. The quenching effect is also true for the 8hydroxyquinoline coordinated $\mathrm{Zn}_{x} \mathrm{Pt}(x \approx 0.7)$ NCs. Although nonfluorescent, these bandgap-staggered NC heterostructures could find applications that utilize charge separation such as photovoltaic devices ${ }^{7}$. Due to the size and shape distribution of the NCs, different surface sites - the corners, edges, sides of crystal planes - would have different coordination affinities and local coordination fields; Second, the NC surface complexes may interact with the surface defect states ${ }^{22}$. Both of them are additional factors that would affect the fluorescent property of the surface complexes. For example, when the quantum dots of CdSe and $\mathrm{CdSe} / \mathrm{CdS}$ are coordinated with 8-hydroxyquinoline, surfacecomplex-related fluorescence is observed (Fig. 5b-e). Although the Förster resonance energy transfer (FRET) rule is satisfied for the energy transfer from the surface complex to the quantum dots, the fluorescence of the quantum dot core is severely quenched. It may due to the surface states and/or the energy level alignment structure of the system which needs further investigation.

Discussion on Type-B Fluorescent Surface Coordination Complexes. It has been shown that, by using proper ligands, the 

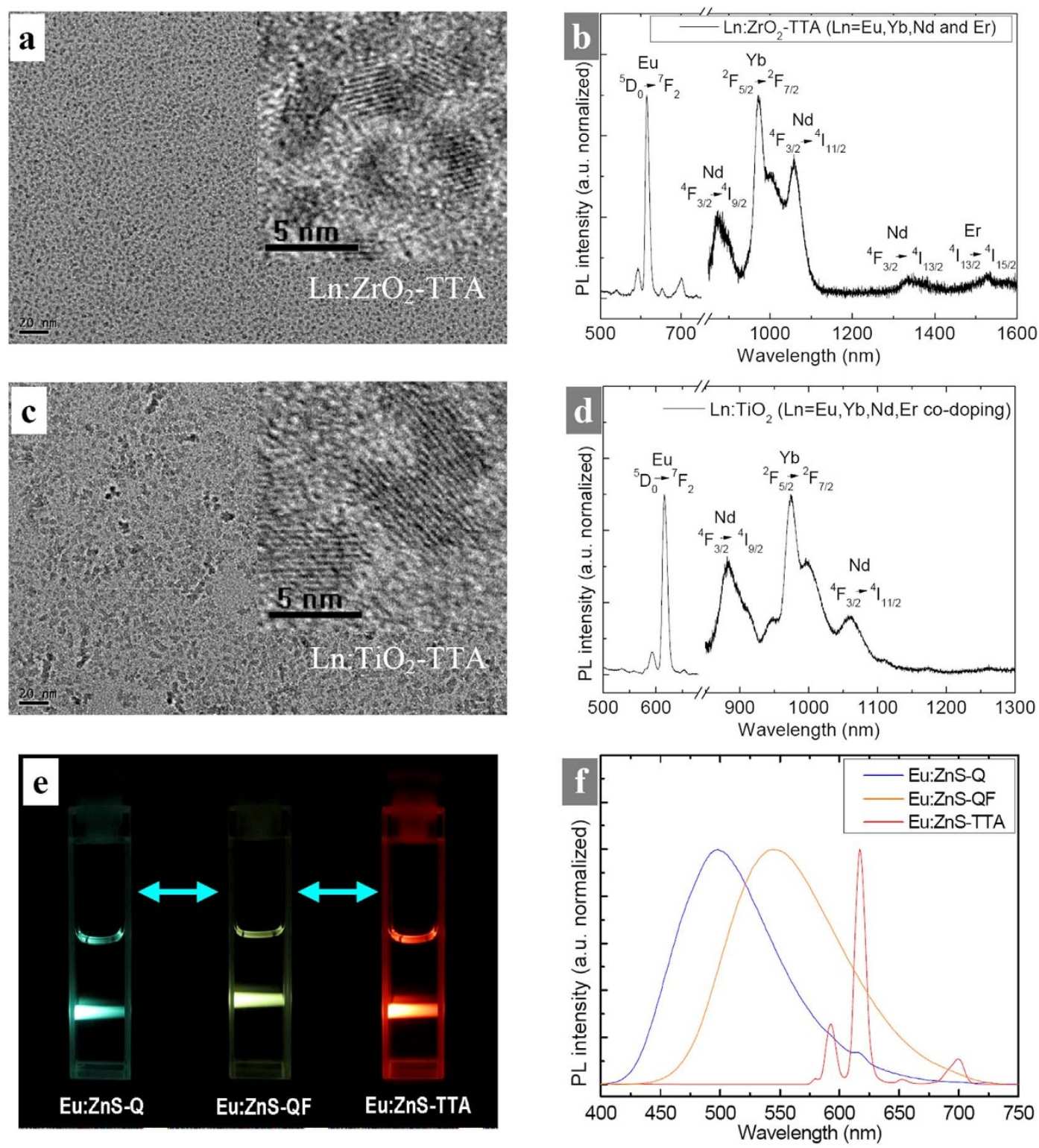

Figure 3 Characterization of multi-color emission NCs and photoluminescence tuning by ligands exchange. (a) TEM image and (b) multi-color photoluminescence spectrum of lanthanide co-doped $\mathrm{Ln}: \mathrm{ZrO}_{2}$-TTA NCs ( $\mathrm{Ln}=\mathrm{Eu}, \mathrm{Yb}, \mathrm{Nd}$ and Er). (c) TEM image and (d) multi-color photoluminescence spectrum of lanthanide co-doped $\mathrm{Ln}_{\mathrm{TiO}}$-TTA NCs ( $\mathrm{Ln}=\mathrm{Eu}, \mathrm{Yb}, \mathrm{Nd}$ and $\mathrm{Er}$ ). (e) Photoluminescence images and (f) spectra of the Eu:ZnS-Q, Eu:ZnS-QF and Eu:ZnS-TTA NCs. The ligands of Q, QF and TTA can be exchanged between each other reversibly, resulting switchable fluorescence. (scale bar $20 \mathrm{~nm}$, inset scale bar $5 \mathrm{~nm}$; Excitation: $360 \mathrm{~nm}$ ).

photoluminescence intensity can be greatly enhanced in type-B fluorescent NCs, with less importance of the host materials of the NCs. Nevertheless, the coordination field or coordination number of a surface complex is determined by both the outer ligand coordination field and the NC crystal field. As a consequence, even for type-B fluorescent NCs with the same centre metal and outer coordination ligand, the fine-structure of the photoluminescence excitation spectra and photoluminescence spectra of the NCs with different NC cores vary from each other. For an example, Fig. S7 shows apparently different photoluminescence excitation (Emission: $615 \mathrm{~nm})$ spectra of the Eu:Au-TTA, Eu:ZnS-TTA, Eu:TiO ${ }_{2}$-TTA and $\mathrm{Eu}: \mathrm{ZrO}_{2}$-TTA NCs; Also their fine-structure of the photoluminescence spectra of the $f-f$ transitions vary: the Eu:ZnSTTA and Eu:ZrO 2 -TTA show stronger ${ }^{5} \mathrm{D}_{0} \rightarrow{ }^{7} \mathrm{~F}_{1}$ transition (magnetic dipole transition) at $\sim 593 \mathrm{~nm}$ than ${ }^{5} \mathrm{D}_{0} \rightarrow{ }^{7} \mathrm{~F}_{4}$ transition (electric dipole transition) at $\sim 700 \mathrm{~nm}$, while Eu:Au-TTA and Eu:TiO 2 -TTA show stronger ${ }^{5} \mathrm{D}_{0} \rightarrow{ }^{7} \mathrm{~F}_{4}$ than ${ }^{5} \mathrm{D}_{0} \rightarrow{ }^{7} \mathrm{~F}_{1}$. For type-B fluorescent NCs, the surface complex structure provides highly asymmetric environment surrounding the lanthanide ion, which also may potentially enhance the photoluminescence ${ }^{29}$. The steric effects introduced by the NC surface and the surface ligands may inhibit solvent molecules or second 'quenching' ligands to coordinate to a single lanthanide ion (e.g., $\mathrm{H}_{2} \mathrm{O}$ or $-\mathrm{OH}$ coordinated to surface ions of $\mathrm{Nd}^{3+}$ or $\mathrm{Er}^{3+}$ ), reducing non-radiative transitions ${ }^{30}$.

Light Emitting Device (LED) Based on NCs with Fluorescent Surface Coordination Complex. The development of organic light emitting devices (OLEDs) has been proven one of the most exciting advances in electronics in the past decade, with fluorescent coordination complexes such as $\mathrm{AlQ}_{3}$ or $\mathrm{ZnQ}_{2}$ being excellent examples of simple complexes exhibiting this property ${ }^{31,32}$. Additionally, many inorganic nanomaterials are also used as nonfluorescent components of electronic devices ${ }^{20,33,34}$. NCs with fluorescent surface complexes can potentially build a bridge 

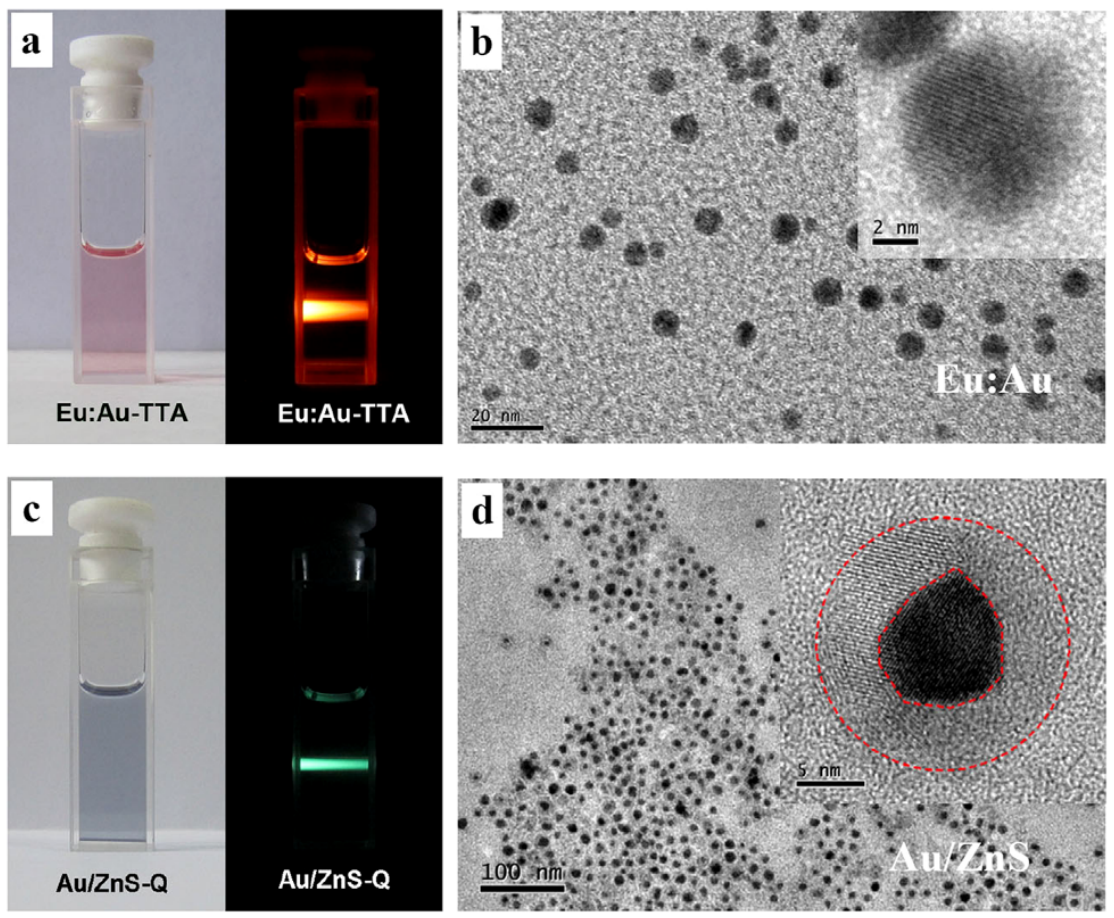

Figure 4 | Fluorescent colloidal NCs structurally containing metal NCs. (a) Daylight and photoluminescence images and (b) TEM image of the Eu:AuTTA NCs (scale bar $20 \mathrm{~nm}$, inset scale bar $2 \mathrm{~nm}$ ). (c) Daylight and photoluminescence images and (d) TEM image of the Au/ZnS-Q NCs (scale bar $100 \mathrm{~nm}$, inset scale bar $5 \mathrm{~nm}$ ). (Excitation: $360 \mathrm{~nm}$ ).

between these two realms of useful inorganic materials. As an example, using ZnS-Q NCs as the active emitter, an electronicallydriven light emitting device was fabricated (Fig. 6a-c, Fig. S8). The good dispersibility of the ZnS-Q NCs in organic solvents makes it feasible to prepare thickness-controllable pure or organic-blended NC films by spin coating or printing techniques; while the solubility of the corresponding complexes of $\mathrm{AlQ}_{3}$ or $\mathrm{ZnQ}_{2}$ is limited and high temperature sublimation in vacuum with expensive equipments is required. For fluorescent quantum dots based $\mathrm{LED}^{3-5}$, surface ligands have to be designed deliberately to optimize the balance between the carrier injection, solubility and the quantum yield ${ }^{19}$, which would not be obstacles for the surface complex fluoresced NCs because the fluorescent surface complex can be intrinsically carrier transportable. These merits deliver new possibilities for fabricating LED devices by simplifying the production process and reducing the cost.

NCs with Fluorescent Surface Coordination Complexes for Cell Imaging. As fluorescent semiconductor NCs, quantum dots have become very popular as fluorophores for biological applications ${ }^{9-11}$. One of the critical requirements is that the fluorescence of quantum dots must be sufficiently strong for imaging or detection after the aqueous transition, bio-transport and tagging processes. Given that well-designed NCs can be swiftly coordinated with appropriate ligands to form fluorescent surface complexes, the fluorescence can be activated by attaching the ligands at any time when needed, thus the above requirement is intrinsically satisfied. To test this concept, low toxicity ZnS-MPA NCs (Fig. 6d, MPA: 3-mercaptopropionic acid) were used to image the U87 MG cells in a two-step method. In the first step, aqueous dispersible ZnS-MPA NCs were incubated with the cells for 24 hours. In the second step, a small quantity of 8 hydroxyquinoline solution was introduced to the cells, forming the ZnS-Q NCs instantly in situ. A remarkable fluorescence enhancement was observed by laser confocal fluorescence microscopy (Fig. 6e,f). The NCs with fluorescent surface complexes could be particularly useful in imaging and labeling live cells due to the highly tunable and switchable photoluminescence from visible to infrared within the therapeutic window and multi-color photoluminescence of $\mathrm{NCs}^{35-37}$.

\section{Conclusion}

In summary, by using the 'Surface Complex on NC' approach, types of NCs have been successfully functionalized with coordinated ligands to tremendously expand the family of fluorescent colloidal NCs. Unlike a metal atom alone, the chemical, physical and biological properties of NCs are much more abundant due to the tailorability of the size, shape, individual/assembly structure, component and surface modification, which would bring about superior properties of the surface complex modified NCs over the common complexes. Due to the infinite of possibilities to combine functional surface coordination complex with the NCs, there is plenty of scope for the future exploration of new colloidal NCs. One can imagine multi-functional colloidal NCs such as magnetic NCs with fluorescent or catalytical surface complexes, bio-friendly NCs with bioactive surface complexes, polymerization of the surface complexes, self-assembly of the NCs with functional surface complexes (e.g., Fig. 2f,g) and so on. We anticipate the development of the NCs with functional surface complexes will bring incredible benefits for both scientific research and real world applications.

\section{Methods}

Instruments. Powder X-ray diffraction (XRD) data were collected on a Bruker D8 Xray diffractometer with $\mathrm{Cu}-\mathrm{K} \alpha$ radiation $(\lambda=1.5406 \AA)$. The Fourier transform infrared spectroscopy (FTIR) spectra were measured by a VECTOR 22 spectrometer with $\mathrm{KBr}$ pellets at a resolution of $2 \mathrm{~cm}^{-1}$. Transmission electron microscopy (TEM) images and the corresponding selected area electron diffraction (SAED) pattern were measured on a JEM-2100 transmission electron microscopy at an acceleration voltage of $200 \mathrm{kV}$. The near infrared photoluminescence spectra were measured with a Zolix Omini- $\lambda 300$ fluorescence spectrophotometer (Xe900 lamp) and DInGaAs 1700 detector. The absorption spectra were measured by a Shimadzu UV-2700 UV-vis spectrophotometer. The photoluminescence emission and excitation spectra were collected by a Hitachi F-4600 fluorescence spectrophotometer.

Nanocrystal synthesis. The $\mathrm{Al}_{2} \mathrm{O}_{3} \mathrm{NCs}$ were synthesized by a modified method from ref. 38. InOOH NWs were synthesized by a previously developed method in ref. 39 . $\mathrm{MgO}, \mathrm{Zn}: \mathrm{MgO}$ and $\mathrm{ZnO}$ NCs were synthesized according to ref. 40. The $\mathrm{Ln}(\mathrm{Ln}=\mathrm{Eu}$, 

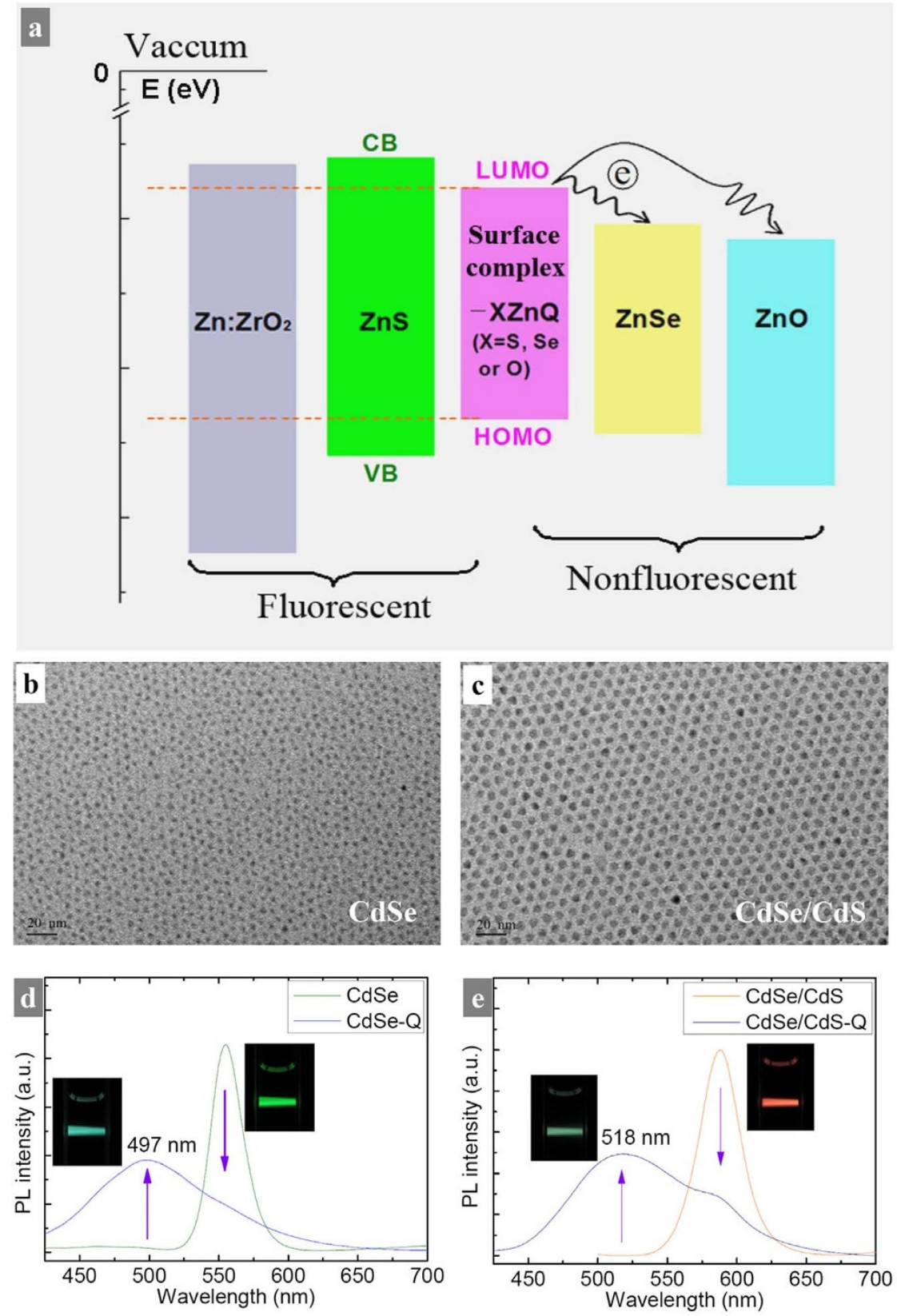

Figure 5 Characterization and energy alignment of surface complex and QDs. (a), A schematic illustration of the proposed energy alignment of the surface complex '-XZnQ' and NCs of $\mathrm{ZnX}\left(\mathrm{X}=\mathrm{S}\right.$, Se or O) and Zn:ZrO ${ }_{2}$. CB: conduction band; VB: valence band. TEM images of (b) CdSe and (c) CdSe/ CdS quantum dots (scale bar: $20 \mathrm{~nm}$ ). Photoluminescence spectra and images of (d) CdSe and CdSe-Q, (e) CdSe/CdS and CdSe/CdS-Q quantum dots.

$\mathrm{Yb}, \mathrm{Nd}$ or/and $\mathrm{Er}$ ) doped/codoped $\mathrm{TiO}_{2} \mathrm{NCs}$ synthesized by a modified method from ref. 41. The $\mathrm{Zn}$ doped and $\mathrm{Ln}\left(\mathrm{Ln}=\mathrm{Eu}, \mathrm{Yb}\right.$, Nd or/and $\mathrm{Er}$ ) doped/codoped $\mathrm{ZrO}_{2} \mathrm{NCs}$ were synthesized by the method of ref. 42 . The ZnSe NCs were synthesized by the method described in ref. 43 . The CdSe and CdSe/CdS(3ML) NCs were synthesized via a previously reported method in ref. 17. The Au/ZnS NCs were synthesized according to ref. 44. Please see "Supporting Information" for the detailed synthesis methods of these NCs and $\mathrm{ZnS}, \mathrm{Ln}\left(\mathrm{Ln}=\mathrm{Eu}, \mathrm{Yb}, \mathrm{Nd}\right.$ or Er) doped $\mathrm{ZnS}, \mathrm{AlOOH}, \mathrm{In}_{2} \mathrm{~S}_{3}$, Eu:Au and $\mathrm{Zn}_{0.7} \mathrm{Pt}$ NCs.

Preparation of NCs with Fluorescent Surface Coordination Complexes. The intended coordinated ligands were modified to the NCs' surface by ligands exchange. A typical ligands exchange procedure: An amount of NCs (e.g. 5-30 mg) is dispersed in $100 \mu \mathrm{l}$ cyclohexane. 2-200 $\mu \mathrm{l}$ of coordinated ligand (Q, QS, QF or TTA) ethanol solution $\left(5 \times 10^{-2}-5 \times 10^{-4} \mathrm{M}\right)$ is added by injection. After seconds of shaking by hand, $0.8 \mathrm{ml}$ ethanol is added to precipitate the NCs. Collected by centrifugation $(8000 \mathrm{rpm}, 1 \mathrm{~min})$, the NCs are washed with cyclohexane and ethanol for a second time to remove any residual ligands or byproducts. The final NCs are re-dispersed and stored in cyclohexane or toluene. For examples, the $\mathrm{MgO}-\mathrm{Q}, \mathrm{Al}_{2} \mathrm{O}_{3}-\mathrm{Q}$ and $\mathrm{ZnS}-\mathrm{Q}$ etc NCs in Fig. 2,3 and Fig. S1-S2, $1 \times 10^{-6}$ mol coordinated ligands were used to exchange with the original ligands of $10 \mathrm{mg}$ of $\mathrm{MgO}, \mathrm{Al}_{2} \mathrm{O}_{3}$ and $\mathrm{ZnS}$ etc $\mathrm{NCs}$, respectively. This process can be applied to the same NCs for several times with different exchanging ligands, indicating the ability of the reversible switch of the fluorescence (e.g. in Fig. 3e,f)

Fabrication of Light Emitting Device Based on NCs with Fluorescent Surface Coordination Complexes (ITO/PEDOT/ZnS-Q:PBD:PVK/TPBi/LiF/Al). Patterned ITO-coated glass slides (15 ohms/sq) were cleaned by sonication in detergent and boiling water, acetone, and alcohol for $30 \mathrm{~min}$, and then plasma cleaned for $5 \mathrm{~min}$. A poly(3,4-ethylenedioxythiophene):poly(styrenesulfonate) (PEDOT) film $(\sim 40 \mathrm{~nm})$ was then spin-coated on top of the ITO surface, dried at $120^{\circ} \mathrm{C}$ for $15 \mathrm{~min}$. The light emitting layer $(80 \mathrm{~nm})$ was deposited by spin coating the solution of ZnS-Q NCs $(20 \mathrm{mg})$, poly $(9$-vinylcarbazole) (PVK) $(8.4 \mathrm{mg})$ and 2-(-4biphenylyl)-5-(4-tert-butylphenyl)-1,2,4-oxadiazole (PBD) $(3.6 \mathrm{mg})$ in $1.2 \mathrm{ml}$ of toluene. In a vacuum chamber at a pressure of $5 \times 10^{-4}$ Torr, 1,3,5-tris (1-phenyl-1Hbenzimidazol-2-yl)benzene (TPBi) $(20 \mathrm{~nm}), \operatorname{LiF}(1.2 \mathrm{~nm})$ and $\mathrm{Al}(80 \mathrm{~nm})$ was thermal evaporated in sequence. The EL spectrum was measured with a spectrometer (PR650) in ambient air condition at room temperature.

Aqueous Transfer of the ZnS NCs. $300 \mathrm{mg}$ of TMAH, $60 \mu \mathrm{l}$ of MPA, $20 \mathrm{mg}$ of ZnS NCs in $0.2 \mathrm{ml}$ of chloroform, $0.4 \mathrm{ml}$ of ethanol and $0.5 \mathrm{ml} \mathrm{H}_{2} \mathrm{O}$ were added to $2 \mathrm{ml}$ of 

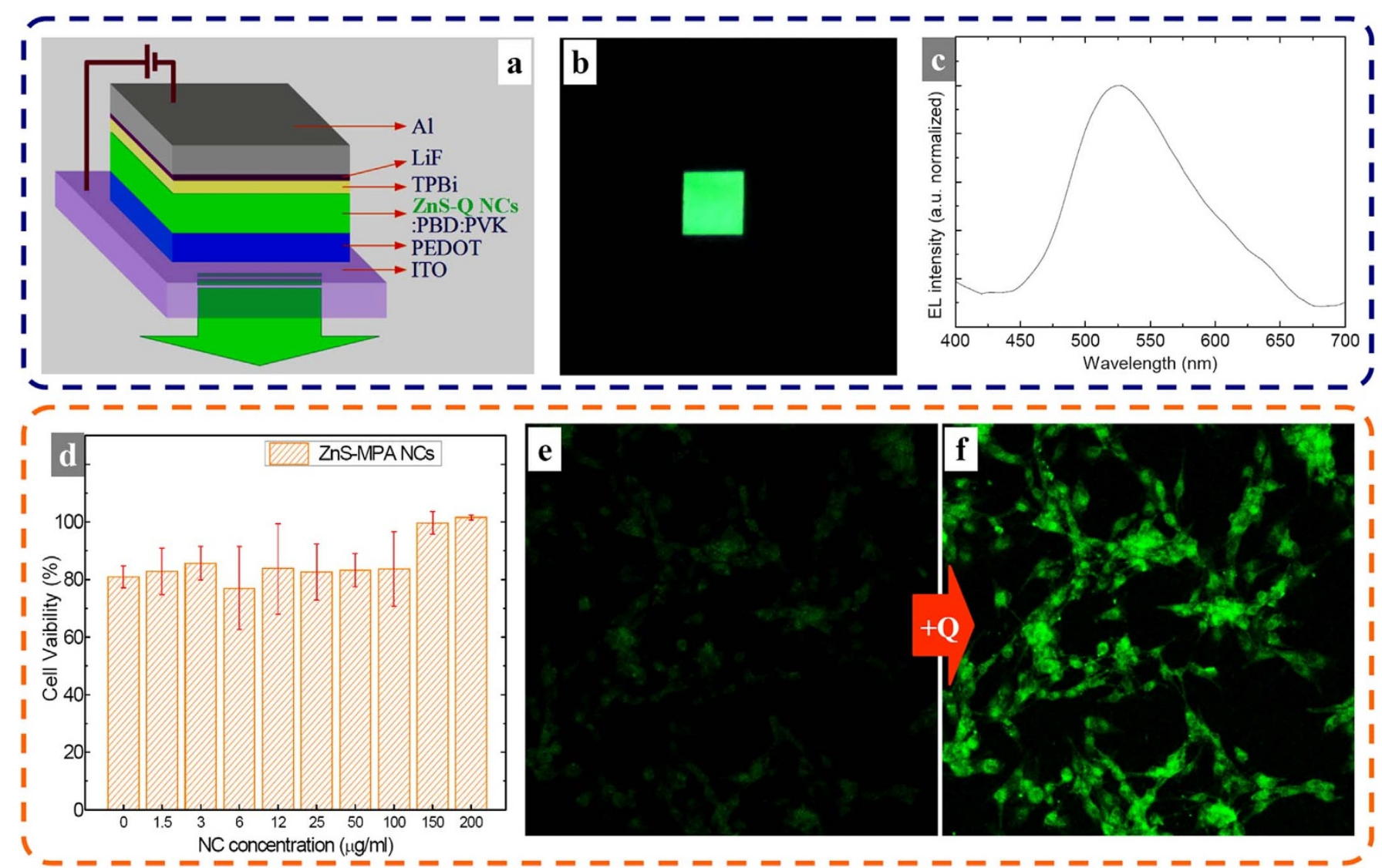

Figure 6 | Applications of the NCs Fluoresced by surface complexes in electronic device and biology. (a) Sketched structure of the ZnS-Q NCs based electro-driven light emitting device. (b) Electroluminescence (EL) image and (c) EL spectrum of the device, operating at 17 V. (d) The cytotoxicity of ZnS-MPA NCs to U87 MG cells after 24-hours exposure to different NCs concentration. It shows no obvious cytotoxicity effect on U87 MG cells. (e) The laser confocal microscopy image of U87 MG cells cultured with the concentration $200 \mu \mathrm{g} / \mathrm{ml}$ of ZnS-MPA NCs for $24 \mathrm{~h}$. (f) The same visual field and observation condition when $10 \mu \mathrm{l}$ 8-hydroxyquinoline solution $\left(5 \times 10^{-4} \mathrm{M}\right)$ was added to from the fluorescent $\mathrm{ZnS}$-Q NCs, lighting the cells instantly. (Excitation: $403 \mathrm{~nm}$; Emission: 506-514 nm).

chloroform sequentially. Thoroughly shaken, the mixture under went a phase separation. The supernatant phase was collected and the lower phase discarded. Ethanol and ethyl acetate were added to the supernatant portion until turning white turbid. The product was separated by centrifugation. The precipitate was washed two times by $\mathrm{H}_{2} \mathrm{O}$, ethanol and ethyl acetate to eliminate the residue MPA, TMAH and byproducts. The final $\mathrm{ZnS}-\mathrm{MPA}$ NCs was redispersed in $\mathrm{H}_{2} \mathrm{O}$ forming a clear solution.

Cell Culture. Human malignant glioma cell line (U87 MG) were cultured in DMEM medium with $10 \% \mathrm{FBS}, 100 \mathrm{U} / \mathrm{ml}$ penicillin, and $100 \mathrm{mg} / \mathrm{ml}$ streptomycin at $37^{\circ} \mathrm{C}$, $5 \% \mathrm{CO}_{2}$-humidified environment of $10^{5} / \mathrm{ml}$ overnight. The cells were incubated with $200 \mu \mathrm{l}$ of $1.5 \mu \mathrm{g} / \mathrm{ml}, 3 \mu \mathrm{g} / \mathrm{ml}, 6 \mu \mathrm{g} / \mathrm{ml}, 12 \mu \mathrm{g} / \mathrm{ml}, 25 \mu \mathrm{g} / \mathrm{ml}, 50 \mu \mathrm{g} / \mathrm{ml}, 100 \mu \mathrm{g} / \mathrm{ml}$ and $200 \mu \mathrm{g} / \mathrm{ml}$ of ZnS-MPA NCs for $24 \mathrm{~h}$, respectively. (Sample number $\mathrm{n}=3$ )

MTT Assay. Thiazolyl blue tetrazolium bromide (MTT) was added to each well of cells (final concentration $0.5 \mathrm{mg} / \mathrm{ml}$ ) and incubated for $4 \mathrm{~h}$ at $37^{\circ} \mathrm{C}$ in a humidified atmosphere of $95 \%$ air, $5 \% \mathrm{CO}_{2}$. DMSO was added to solubilize the blue MTTformazan product and the sample was incubated for a further $30 \mathrm{~min}$ at room temperature. Absorbance of the solution was read at a test wavelength of $490 \mathrm{~nm}$. The result shows the cytotoxicity of U87 MG cells after 24 h exposure to different concentration of ZnS-MPA NCs and it didn't have any obvious affect on U87 MG up to the concentration of $200 \mu \mathrm{g} / \mathrm{ml}$ (Fig. 6d)

Cell Imaging. U87 MG cells were cultured in 6-well plates with the concentration $200 \mu \mathrm{g} / \mathrm{ml}$ of NCs for $24 \mathrm{~h}$ and observed with the laser confocal microscopy (Nikon Ti-E, Japan). Then $10 \mu \mathrm{l}$ Q solution (10\% vol ethanol aqueous solution with the concentration of $5 \times 10^{-4} \mathrm{M}$ ) was added and followed by rinsing with water. Instant fluorescence was observed from the ZnS-Q NCs labeled U87 MG cells.

1. Alivisatos, A. P. Semiconductor clusters, nanocrystals, and quantum dots. Science 271, 933-937 (1996).

2. Talapin, D. V., Lee, J.-S., Kovalenko, M. V. \& Shevchenko, E. V. Prospects of colloidal nanocrystals for electronic and optoelectronic applications. Chem. Rev. 110, 389-458 (2010).
3. Tan, Z. A. et al. Bright and color-saturated emission from blue light-emitting diodes based on solution-processed colloidal nanocrystal quantum dots. Nano Lett. 7, 3803-3807 (2007).

4. Tan, Z. A. et al. Colloidal nanocrystal-based light-emitting diodes fabricated on plastic toward flexible quantum dot optoelectronics. J. Appl. Phys. 105, 034312 (2009).

5. Tan, Z. A. et al. Near-band-edge electroluminescence from heavy-metal-free colloidal quantum dots. Adv. Mater. 23, 3553-3558 (2011).

6. Semonin, O. E. et al. Peak external photocurrent quantum efficiency exceeding $100 \%$ via MEG in a quantum dot solar cell. Science 334, 1530-1533 (2011).

7. Kramer, I. J. \& Sargent, E. H. Colloidal quantum dot photovoltaics: a path forward. Acs Nano 5, 8506-8514 (2011).

8. Ip, A. H. et al. Hybrid passivated colloidal quantum dot solids. Nature nanotech. 7, 577-582 (2012).

9. Bruchez, M., Moronne, M., Gin, P., Weiss, S. \& Alivisatos, A. P. Semiconductor nanocrystals as fluorescent biological labels. Science 281, 2013-2016 (1998).

10. Chan, W. C. W. \& Mnie, S. Quantum dot bioconjugates for ultrasensitive nonisotopic detection. Science 281, 2016-2018 (1998).

11. Medintz, I. L., Uyeda, H. T., Goldman, E. R. \& Mattoussi, H. Quantum dot bioconjugates for imaging, labelling and sensing. Nature Mater. 4, 435-446 (2005).

12. Peng, X. G. et al. Shape control of CdSe nanocrystals. Nature 404, 59-61 (2000).

13. Yin, Y. D. \& Alivisatos, A. P. Colloidal nanocrystal synthesis and the organicinorganic interface. Nautre 437, 664-670 (2005).

14. Wang, X., Zhuang, J., Peng, Q. \& Li, Y. D. A general strategy for nanocrystal synthesis. Nature 437, 121-124 (2005).

15. Alivisatos, A. P. et al. Organization of 'nanocrystal molecules' using DNA. Nature 382, 609-611 (1996).

16. Shevchenko, E. V., Talapin, D. V., Kotov, N. A., O’Brien, S. \& Murray, C. B. Structural diversity in binary nanoparticle superlattices. Nature 439, 55-59 (2006).

17. Xu, X. X. et al. Assembly and separation of semiconductor quantum dot dimers and trimers. J. Am. Chem. Soc. 133, 18062-18065 (2011). 
18. Xu, X. X., Ji, J. W., Wang, G. \& You, X. Z. Exciton coupling of surface complexes on a nanocrystal surface. ChemPhysChem DOI: 10.1002/cphc.201402156.

19. Milliron, D. J., Alivisatos, A. P., Pitois, C., Edder, C. \& Fréchet, J. M. J. Electroactive surfactant designed to mediate electron transfer between CdSe nanocrystals and organic semiconductors. Adv. Mater. 15, 58-61 (2003).

20. Talapin, D. V. \& Murray, C. B. PbSe nanocrystal solids for $\mathrm{n}$ - and p-channel thin film field-effect transistors. Science 310, 86-89 (2005).

21. Kovalenko, M. V., Scheele, M. \& Talapin, D. V. Colloidal nanocrystals with molecular metal chalcogenide surface ligands. Science 324, 1417-1420 (2009).

22. Cordones, A. A., Scheele, M., Alivisatos, A. P. \& Leone, S. R. Probing the interaction of single nanocrystals with inorganic capping ligands: time-resolved fluorescence from cdse-cds quantum dots capped with chalcogenidometalates. J. Am. Chem. Soc. 134, 18366-18373 (2012).

23. Demas, J. N. \& DeGraff, B. A. Design and applications of highly luminescent transition metal complexes. Anal. Chem. 63, 829A-837A (1991).

24. Mokari, T., Rothenberg, E., Popov, I., Costi, R. \& Banin, U. Selective growth of metal tips onto semiconductor quantum rods and tetrapods. Science 304, 1787-1790 (2004)

25. Wang, C., Xu, C. J., Zeng, H. \& Sun, S. H. Recent progress in syntheses and applications of dumbbell-like nanoparticles. Adv. Mater. 21, 3045-3052 (2009).

26. Aslan, K., Wu, M., Lakowicz, J. R. \& Geddes, C. D. Fluorescent core-shell Ag@ $\mathrm{SiO} 2$ nanocomposites for metal-enhanced fluorescence and single nanoparticle sensing platforms. J. Am. Chem. Soc. 129, 1524-1525 (2007).

27. Liu, N. G., Prall, B. S. \& Klimov, V. I. Hybrid gold/silica/nanocrystal-quantum-dot superstructures: synthesis and analysis of semiconductor-metal interactions. J. Am. Chem. Soc. 128, 15362-15363 (2006).

28. Hopkins, T. A. et al. Substituted aluminum and zinc quinolates with blue-shifted absorbance/luminescence bands: synthesis and spectroscopic, photoluminescence, and electroluminescence characterization. Chem. Mater. 8 , 344-351 (1996).

29. Iwamuro, M. et al. Photosensitized luminescence of neodymium(III) coordinated with 8-quinolinolates in DMSO- $d_{6}$. Bull. Chem. Soc. Jpn. 73, 1359-1363 (2000).

30. $\mathrm{Yu}, \mathrm{R}$. B. et al. $\mathrm{Nd}_{2} \mathrm{O}_{3}$ nanoparticles modified with a silane-coupling agent as a liquid laser medium. Adv. Mater. 19, 838-842 (2007).

31. Evans, R. C., Douglas, P. \& Winscom, C. J. Coordination complexes exhibiting room-temperature phosphorescence: evaluation of their suitability as triplet emitters in organic light emitting diodes. Coordin. Chem. Rev. 250, 2093-2126 (2006).

32. Burn, P. L., Lo, S. C. \& Samuel, I. D. W. The development of light-emitting dendrimers for displays. Adv. Mater. 19, 1675-1688 (2007).

33. Yu, D., Wang, C. \& Guyot-Sionnest, P. $n$-Type conducting CdSe nanocrystal solids. Science 300, 1277-1280 (2003).

34. Zhang, D. Q. et al. Synthesis of ultralong copper nanowires for high-performance transparent electrodes. J. Am. Chem. Soc. 134, 14283-14286 (2012).

35. Han, M. Y., Gao, X. H., Su, J. Z. \& Nie, S. M. Quantum-dot-tagged microbeads for multiplexed optical coding of biomolecules. Nat. Biotechnol. 19, 631-635 (2001).

36. Welsher, K. et al. A route to brightly fluorescent carbon nanotubes for nearinfrared imaging in mice. Nat. Nanotechnol. 4, 773-780 (2009).

37. Zhang, Y. et al. $\mathrm{Ag}_{2} \mathrm{~S}$ quantum dot: a bright and biocompatible fluorescent nanoprobe in the second near-infrared window. ACS Nano 6, 3695-3702 (2012).
38. Zhou, S. X., Antonietti, M. \& Niederberger, M. Low-temperature synthesis of $\gamma$ alumina nanocrystals from aluminum acetylacetonate in nonaqueous media. Small 3, 763-767 (2007).

39. Xu, X. X. \& Wang, X. Size-and surface-determined transformations: from ultrathin InOOH nanowires to uniform $c$ - $\mathrm{In}_{2} \mathrm{O}_{3}$ nanocubes and $r h$ - $\mathrm{In}_{2} \mathrm{O}_{3}$ nanowires. Inorg. Chem. 48, 3890-3895 (2009).

40. Yang, Y. F. et al. Dopant-induced shape evolution of colloidal nanocrystals: the case of zinc oxid. J. Am. Chem. Soc. 132, 13381-13394 (2010).

41. Li, X. L., Peng, Q., Yi, J. X., Wang, X. \& Li, Y. D. Near monodisperse $\mathrm{TiO}_{2}$ nanoparticles and nanorods. Chem. Eur. J. 12, 2383-2391 (2006).

42. Xu, X. X. \& Wang, X. Fine tuning of the sizes and phases of $\mathrm{ZrO}_{2}$ nanocrystals. Nano Res. 2, 891-902 (2009).

43. Viswanatha, R., Brovelli, S., Pandey, A., Crooker, S. A. \& Klimov, V. I. Copperdoped inverted core/shell nanocrystals with "permanent" optically active holes. Nano Lett. 11, 4753-4758 (2011).

44. Zhang, J. T., Tang, Y., Lee, K. \& Ouyang, M. Nonepitaxial growth of hybrid coreshell nanostructures with large lattice mismatches. Science 327, 1634-1638 (2010).

\section{Acknowledgments}

This work as supported by the Major State Basic Research Development Program of China (Grant No. 2013CB922102, 2011CB808704), the National Natural Science Foundation of China (Grant No. 91022031, 21301089) and Jiangsu Province Science Foundation for Youths (BK20130562). The authors gratefully acknowledge J. E. Beves for useful advice and discussions on the manuscript.

\section{Author contributions}

X.X. designed the experiments and wrote the manuscript. G.W., J.J. \& X.X. performed most of the experiments, assisted by X.Y.; G.W. \& J.J. contributed equally. X.Z. did the LED device fabrication. Y.Z. and Q.W. did the cell imaging. All authors discussed the results and commented on the manuscript.

\section{Additional information}

Supplementary information accompanies this paper at http://www.nature.com/ scientificreports

Competing financial interests: The authors declare no competing financial interests.

How to cite this article: Wang, G. et al. Colloidal Nanocrystals Fluoresced by Surface Coordination Complexes. Sci. Rep. 4, 5480; DOI:10.1038/srep05480 (2014).

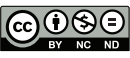

This work is licensed under a Creative Commons Attribution-NonCommercialNoDerivs 4.0 International License. The images or other third party material in this article are included in the article's Creative Commons license, unless indicated otherwise in the credit line; if the material is not included under the Creative Commons license, users will need to obtain permission from the license holder in order to reproduce the material. To view a copy of this license, visit http:// creativecommons.org/licenses/by-nc-nd/4.0/ 